\title{
Improving anthropomorphic robot stability using advanced intelligent control interfaces
}

\author{
Vladareanu Luige $^{1}$, Pandelea Marius ${ }^{1}$, Vladareanu Victor $^{1}$, Feng Yongfei ${ }^{2}$, \\ Grosu Vlad Alexandru ${ }^{3}$, El Emary Ibrahiem ${ }^{4}$, Moga Daniel $^{5}$, Iliescu Mihaiela ${ }^{1}$ \\ ${ }^{1}$ Romanian Academy, Institute of Solid Mechanics, Bucharest, Romania \\ ${ }^{2}$ Ningbo University, Ningbo, China \\ ${ }^{3}$ Politehnica University of Bucharest, Romania \\ ${ }^{4}$ King Abdulaziz University Jeddah, Computer Science and Systems, Saudi Arabia \\ ${ }^{5}$ Technical University of Cluj-Napoca, Romania
}

\section{Article Info}

Received Dec 13, 2018

\begin{abstract}
The article focused on the advanced intelligent control of the stability of anthropomorphic walking robots (AWRs), in order to validate a new and useful method of moving in the virtual environment, which determines a substantial increase in their stability. The obtained results lead to Versatile Intelligent Portable Robot Platform VIPRO, developed to improve the walking anthropomorphic robots' performances, provide unlimited power for design, test, experiment the real time control methods by integrating the Intelligent Control Interfaces (ICIs) in robot modeling and simulation for all types of humanoid robots, rescue robots, firefighting robots.
\end{abstract}

Intelligent Control Interfaces

Robotics

Anthropomorphic robots

Robot stability

Real time control systems

\section{Corresponding Author:}

Pandelea Marius,

Romanian Academy, Institute of Solid Mechanics, 15 Constantin Mille street, 010141 Bucharest, Romania.

Email:marpius65@yahoo.com

\section{Introduction}

Today, humans' life seems unimaginable without artificial intelligence that is present in real life in manufacturing processes, cars etc., in areas of activity applied in mathematics complex calculus, informatics, robotics, medicine, agriculture, economics, communications, business, thanks to the many advantages offered. The complex process of developing artificial intelligence is a space-temporal one, in which the continuous transfer of data and information from the working environment is up taken by controllers [2], [9], so that AWRs find their balance progressively.

The robots and robotics must provide support and friendship to humans. The relationship between humans and robots must be based on communication and realistic expectations, which means knowing the robot by the human and the anticipation of human reactions by the robot [5]. The benefits of robots [20], [21] have been proved to be spectacular, especially by precision, resistance, risk-free work in hazardous environments, adaptation, flexibility, improving the qualities of activities, high capacity to perform tasks and last but not least, increased economic efficiency.

AWR, this complex and sophisticated mechatronic entity, already has a huge influence on Earth's civilization. Speaking in a physical and intellectual sense, AWR body is still developing and growing, in relation to human's needs and dreams. Absolutely all parts and subsystems embedded in AWR structure are subjected to thorough research, with acute need for intellectual creative development in the smallest details. These include 
materials, parts manufacturing technology, sensors, actuators, internal electrical circuits and communication networks, algorithms and software etc. [9].

AWR, like humans, should be given the ability to be self-controlled and auto developed through understanding, learning, adapting, experience and not least, prediction, associated with a mindset and rational behavior, without basically major differences between the human brain and the artificial brain [5]. AWR control can be achieved either through intelligent strategies, techniques and methods, superior to existing ones or through remodeling of the existing working environment. In order to achieve a high level of AWR control, researchers need to know and understand the current state of the realm; they must develop new performance subsystems that ensure with acceptable errors the actions envisaged, validated in the real environment.

The main issues of topical use of AWR consist of precise identification of the location, elimination of legs slipping, contact with the travel field, positioning and sensor number install, pragmatic platforms for simulation and research, testing and experimentation. The development of AWR controllers requires new strategies and intelligent advanced control methods to cope with adaptability AWR to environment and workspace.

This article is focused on the intelligent advanced control of AWR stability, integrated as an intelligent control interface in the Versatile Intelligent Portable Robot Platform VIPRO for design, testing, simulation and validation of robot performance. The proposed study includes the current state of research, new adopted solutions, proposed hybrid techniques and methods that result in a substantial increase in AWR stability control, a case study and the results of research experiments after the simulation computer.

\section{Ishikawa Diagram (cause and effect diagram)}

The control of balance stability is one of the of researchers, scientists and engineer's "obsession", and, in this regard, many solutions have been developed. In the state of rest, as well as during the journey, after a special effort, AWR must permanently maintain its balance to avoid harmful actions.

Ishikawa diagram, known as the „cause-and-effect diagram” or the „Fishbone diagram”, was created by Kaoru Ishikawa in 1943 and graphically represents the possible causes of a given effect. Conceptual diagram is one of the 7 quality tools that shows the relationship of all factors (causes) leading to a given situation (effect). The diagram identifies some major causes and decomposes them in subsequent sub-causes and sub-divisions. It is a powerful tool that can be used to analyze and solve problems.

In order to achieve the intelligent advanced control of AWR stability, integrated as an intelligent control interface in the Versatile Intelligent Portable Robot Platform VIPRO [19], [21], it is presented, shortly, the image of the current situation in the field of control of the stability of walking robots, gathering some of the latest research [11].

In fact, there are considered movements on inclined terrain [4], [7], [12], [13], [14], [16], [24], [25] which by means of an explicit trajectory planning [8], [10], [13] with a strategy based on the ZMP criterion [4], [8], [10], [12], [13], [16], [23], [25] or CPG [24], where a careful analysis of the robot's step [4], [8], [10], [13], [14], [16], [23], [24], [25] and in which the inertia [4], [7], [8], [14], [16], [25] of the various components of the robot have not been avoided [15]. The control methods are applied to the CoM center of mass [4], [7], [8], [10], [12], [13], [16], [24], [25] or to the CoG center of gravity [10], [14], the difference between the positions of the two points are not significant in an uniform gravitational field. The control methods are based on the model of the linear inverted pendulum LIP [4], [7], [8], [13], [23], [25] and use neural networks [6], [16], [24], fuzzy logic [7], [12] predictive [8], balance [4], [7], [8], [10], [12], [13], [16], [24] or energy efficiency calculation [14], [16], [26]. The proposed theoretical results were simulated in the virtual environment [12], [16], [24], [25] with the Webots simulation software [13], [16] or with the application Choregraphe [23] and tested in the real environment on the Nao robot [8], [13], [16], [23], [25] or other models of walking robots [4], [7], [10], [12], [24]. Stability control was carried out either online [10], [12] or offline [1], [25]. 


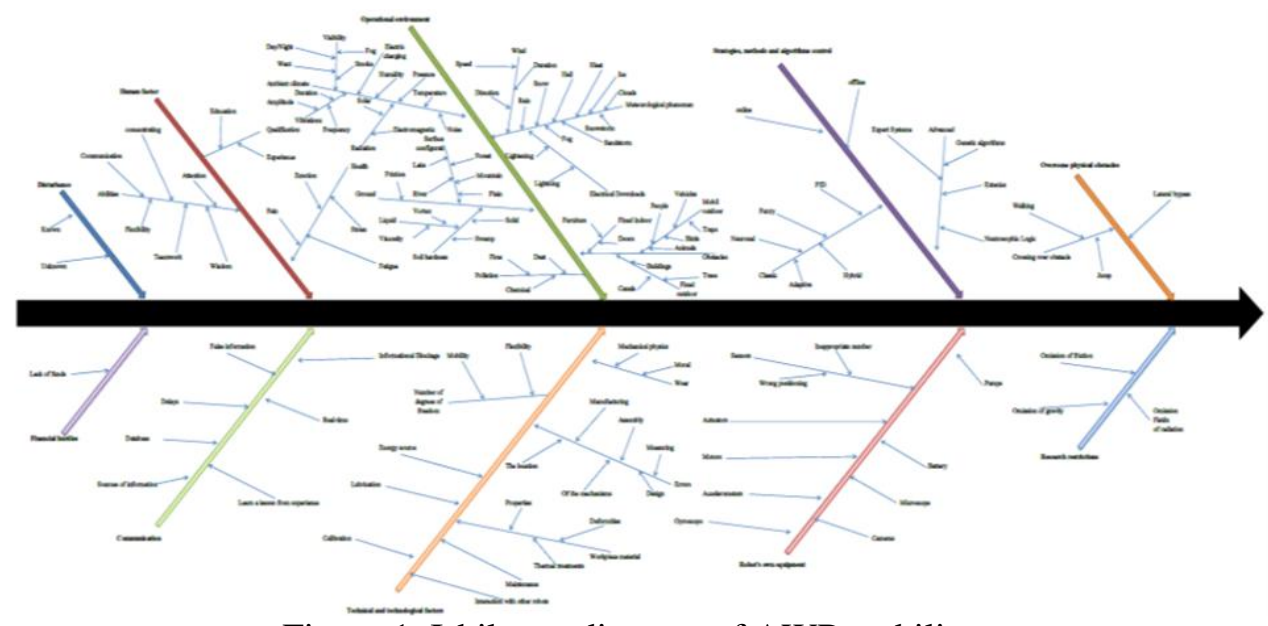

Figure 1. Ishikawa diagram of AWR stability

The Ishikawa diagram of AWR stability (Figure 1) integrates the components resulting from the analysis of AWR movement on uneven, unstructured and obstructed terrain, to which two first-rank components are added, i.e. the study of the static balance by CoM trajectory [4], [7], [8], [10], [12], [13], [16], [24], [25] and the investigation of the dynamic balance by the trajectory of ZMP [4], [8], [10], [12], [13], [16], [23], [25], as our synthesis above reveals.

It is also demonstrated that high static stability ensures a slow shift, but cannot meet a high-speed requirement. The dynamic stability of the anthropomorphic robotic platform balance is ensured in the conditions of the projection of the ZMP in the support range defined by the AWR legs [11], [13] and is more difficult to maintain than in the case of multiple legs robots.

The errors generated by the AWR modeling, by theoretical calculation reported to the effects and working environment in the control of walking on a horizontal or inclined plane, were included in the Ishikawa diagram based on strategies, methods and algorithms, either by study in plan horizontally and then extrapolate on the slope, or through the reverse linear inverted method pendulum (LIMP) [4], [7], [8], [13], [23], [25], dual-length linear inverted method pendulum (DLLIMP), virtual support point (VSP), energy efficiency [14], [16], [26] dynamic model adopted, sensory analysis of motion robot on unstructured and uneven terrain [11], [17], [18], [19], [21], [22].

The proposed method offers a stable walk with high speed for the NAO robot only in the conditions of a relatively straight route, with no sharp changes in direction. In [25], Yuan et al. applies the 3D LIMP for offline generation of walking stepped on a known fixed angle slope and for obtaining the trajectory of CoM. The movement of the stepping robot is controlled by the position at the leg landing, the inverse kinematical D$\mathrm{H}$ of the leg parameters, a Kalman type filter and the inclusion of a inverse kinematic controller for the correction of the CoM position in real time. Performed experiments showed that the method proposed with closed loop induces a good control of stability over long distances with a minor deviation on the move. In [16] the autors propose neural network controllers to optimize the motion in the sagittal and lateral plan of the robot in the conditions of a low-energy consume walking, without a support area and when the ankle of the leg can rotate freely around the wrist, while it is on the ground and the method for controlling the dynamic walking of AWR on the unknown terrain, which have been evaluated on the Nao robot. In [13], Shah analyzes the stability of the Nao robot walking on surfaces with different slopes in the conditions of a dynamic planning based on the ZMP criterion, a control with open loop and simulation in the virtual environment with the Webots software.

The result of these studies, analyses and research was the basis for the realization of the Ishikawa diagram of AWR stability (Figure 1), which led to development and design of the advanced intelligent control interfaces for improve AWR stability, integrated in the Versatile Intelligent Portable Robot Platform VIPRO.

\section{VIPRO Concept for Developing the AWR Intellıgent Control Interfaces}

The Versatile Intelligent Portable Robot Platform VIPRO allows the development of Intelligent Control Interfaces (ICIs) of AWR by providing a design, testing, simulation and validation of intelligent interface realtime control using the virtual projection method [11], [17], [18], [21], [22], in order to improve stability performance. 
Based on the model developed by George Gerbner regarding the importance of communication and perception, the technique required is included in the general AWR control scheme shown below (Figure 2), in order to increase the data and essential information from the workspace, but in combination with the Pareto principle (80/20 rule), which indicates that approximately $80 \%$ of the effects are produced by only $20 \%$ of the causes.

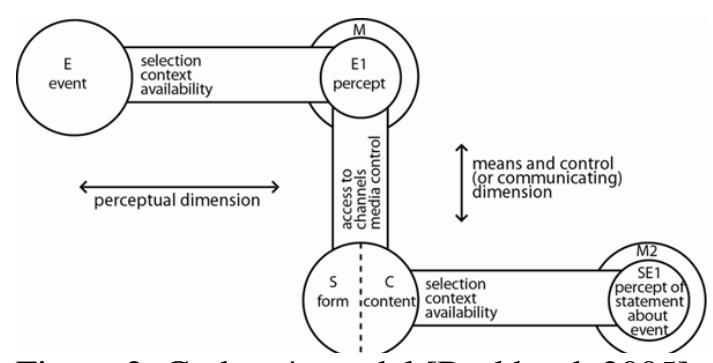

Figure 2. Gerbner's model [Burkhard, 2005]

By applying the Ishikawa diagram on the stability of AWR and the Pareto principle, was created the control system with open architecture for improved stability, integrated in the Inteligent Control Interface AWR (AWR ICIs), presented in the figure 3. The Versatile Intelligent Portable Robot Platform VIPRO [11], [17], [18], [19], [21], [22] was developed to improve the AWR performances, provide unlimited power for design, test, experiment the real time, control methods by integrating the intelligent control interfaces (ICIs) in robot modeling and simulation for all types of humanoid robots, rescue robots, firefighting robots.

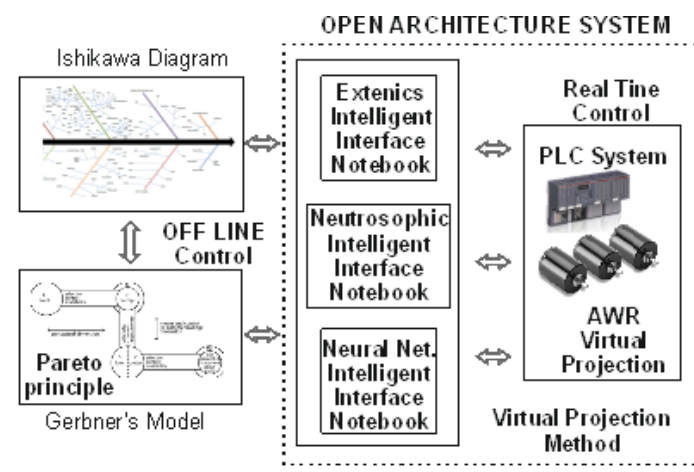

Figure 3. Inteligent interfaces of AWR stability control in open architecture structure (AWR ICIs)

The current VIPRO platform (Figure 4) integrates the Ishikawa diagram and the Pareto principle as two used offline modules in the design phase of the intelligent control interface for robot modeling.

So, in designing the inteligent interface of AWR stability control in the structure with open architecture (AWR ICIs) were identified and used only the sensors, actuators and status parameters applying the Pareto principle and eliminating the Ishikawa diagram events that could influence the stability of AWR below $0.1 \%$.

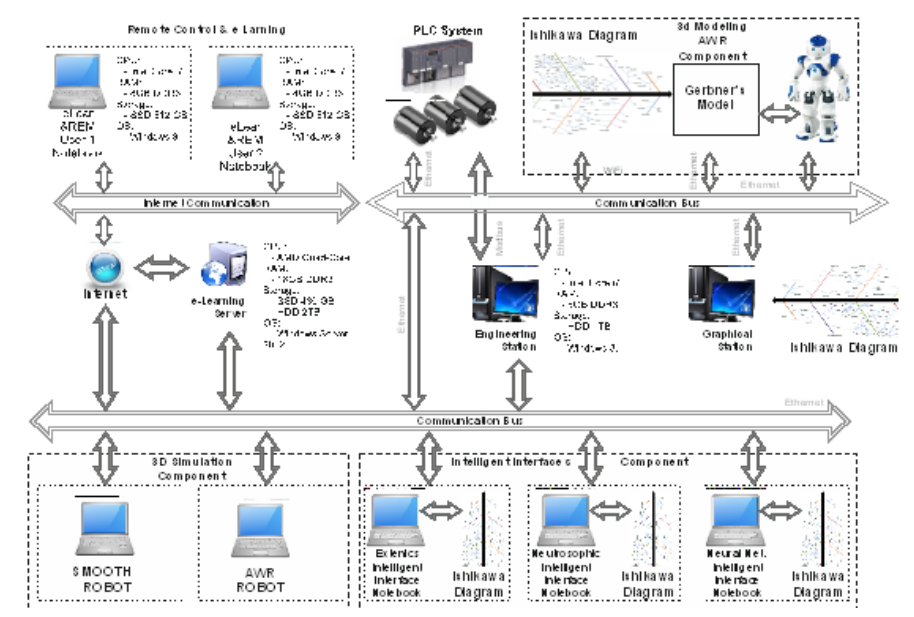


Figure 4. Versatile Intelligent Portable Robot Platform VIPRO

The obtained results lead to the architecture of the Versatile Intelligent Portable Robot VIPRO Platform presented in figure 4.

\section{Conclusions}

The intelligent control interfaces with advanced control technologies adapted to AWR stability were analyzed using the 3D mapping of the environment, human adaptive mechatronics intelligent control interfaces, the online cooperative 3D SLAM intelligent control interface, Robot Neutrosophic intelligent control and extended control through the Extenics control logic based on the Ishikawa diagram.

AWR problems are today of major interest, they are not missing from any agenda of major universities or research centres; there are continuously transmitted messages at international level to speed up their resolution. Only through a full approach, integrated into the real environment can be guaranteed the succes of AWR control. If sometimes the results obtained by robots have been successful, reaching the level of expectations or even passing over the limits imposed by those who gave them life, the human capacities have not been reached yet. The identification of strategic directions that will be followed through the development of the VIPRO platform will lead to new methods of control to improve the stability of AWR.

Experimental results have proved to be efficient and validate the proposed method in different working environments and recommend it in order to improve the stability of AWR.

\section{Acknowledgements}

This work was supported by a grant of the Romanian Ministry of Research and Innovation, CCCDIUEFISCDI, MULTIMOND2 project number PN-III-P1-1.2-PCCDI2017-0637/33PCCDI/01.03.2018, and by KEYTHROB project, number PN-III-P3-3.1-PM-RO-CN-2018-0144 / 2 BM / 2018, within PNCDI III, and by the European Commission Marie Skłodowska-Curie SMOOTH project, Smart Robots for Fire-Fighting, H2020-MSCA-RISE-2016-734875. The authors gratefully acknowledge the support of the Robotics and Mechatronics Department, Insitute of Solid Mechanics of the Romanian Academy.

\section{References}

[1] C. Barz, C. Oprea, Z. Erdei, A. Pop Vadean, "The control of an industrial process with PLC," International Conference on Applied and Theoretical Electricity (ICATE), 2014, https://iopscience.iop.org/article/10.1088/1757-899X/144/1/012006.

[2] M. Boscoianu, G. Prelipcean, M. Lupan, "Innovation enterprise as a vehicle for sustainable developmentA general framework for the design of typical strategies based on enterprise systems engineering, dynamic capabilities, and option pricing," Journal of Cleaner Production, vol. 172, pp. 3498-3507, 2018.

[3] R. A. Burkhard, "Knowledge Visualization: The Use of Complementary Visual Representations for the Transfer of Knowledge. A Model, a Framework, and Four New Approaches," A dissertation submitted to the Swiss Federal Institute of Technology Zurich for the degree of Doctor of Sciences, 2005.

[4] L. Colasanto, N. Perrin, N. Tsagarakis, D. Caldwell, "Dynamically transitioning between surfaces of varying inclinations to achieve uneven-terrain walking," IEEE International Conference on Robotics and Automation (ICRA), May 2014, Hong Kong, China. pp. 210 - 215.

[5] Y. Feng, H. Wang, H. Yan, X. Wang, Z. Jin, L. Vladareanu, "Research on Safety and Compliance of a New Lower Limb Rehabilitation Robot," Journal of Healthcare Engineering, vol. 2017, https://www.hindawi.com/journals/jhe/2017/1523068/abs/, ID 1523068.

[6] M. Iliescu, V. Vladareanu, M. Șerbănescu, M. Lazăr, "Sensor Input Learning for Time-of-Flight Scan Laser," CEAI, vol. 19, no. 2, pp. 51-60, ISSN 1454-8658. 
[7] C. H. Kuo, F. Zal, S. L. Wu, "Development of Fuzzy Logic Controllers for Controlling Bipedal Robot Locomotion on Uneven Terrains with IMU Feedbacks," Indian Journal of Science and Technology, vol. 9(28), DOI: 10.17485/ijst/2016/v9i28/98449, July 2016.

[8] F. H. S. Martínez, F. S. Martínez, E. G. Jacinto, "A Dynamic Walking Strategy for the Nao Robot Considering Path Planning, CoM and ZMP," International Journal of Engineering and Technology (IJET), vol. 10, no. 5, Oct-Nov 2018, DOI: 10.21817/ijet/2018/v10i5/181005021, pp. 1293-1301.

[9] M. A. Moisescu, I. S. Sacala, "Towards the development of interoperable sensing systems for the future enterprise," Journal of Intelligent Manufacturing, 27(1), pp. 33-54, 2016.

[10] K. Nishiwaki, S. Kagami, "Simultaneous Planning of CoM and ZMP based on the Preview Control Method for Online Walking Control," 11th IEEE-RAS International Conference on Humanoid Robots, October 26-28, 2011, pp.745-751.

[11] M. Pandelea, L. Vladareanu, M. Iliescu, R. I. Munteanu, M. Radulescu, "Intelligent Advanced Control Strategies for Mobile Autonomous Robots Stability Through Versatile, Intelligent, Portable VIPRO Platform," The 42th International Conference on ICMSAV, 25-26.10.2018. pp.184, ISSN 2457-8541, ISSN-L 2457-8541.

[12] U. Seven, T. Akbas, K. C. Fidan, M. Yilmaz, K. Erbatur, "Humanoid Robot Walking Control on Inclined Planes," 2011 IEEE International Conference on Mechatronics, DOI: 10.1109/ICMECH.2011.5971237.

[13] B. C. Shah, "Walking on different slopes for Nao humanoid robot for an open loop design," International Journal Of Applied Research In Science And Engineering, International Conference on Emerging Technologies in Engineering, Biomedical, Medical and Science, ETEBMS - July 2017, ISSN (Online): 2456-124X.

[14] A. Smyrli, G. A. Bertos, E. Papadopoulos, "Efficient stabilization of zero-slope walking for bipedal robots following their passive fixed-point trajectories," 2018 IEEE International Conference on Robotics and Automation (ICRA), May 21-25, 2018, Brisbane, Australia.

[15] I. Staretu, S. Jitariu, "Reconfigurable Anthropomorphic Gripper with Three Fingers: Synthesis, Analysis, and Simulation", Applied Mechanics and Materials, vol. 762, pp. 75-82, 2015.

[16] Z. Sun, N. Roos, "Dynamically stable walk control of biped humanoid on uneven and inclined terrain," Neurocomputing, vol. 280, 2018, pp.111-122. https://doi.org/10.1016/j.neucom.2017.08.077.

[17] L. Vladareanu, L. M. Velea, R. I. Munteanu, A. Curaj, S. Cononovici, T. Sireteanu, L. Capitanu, M. S. Munteanu, "Real time control method and device for robots in virtual projection," Patent OSIM 123527/30.04.2013, EP 2105 263, Application number: EP 0946 4001, 2009.

[18] L. Vladareanu, V. Vladareanu, P. Șchiopu, "Hybrid Force-Position Dynamic Control of the Robots Using Fuzzy Applications," 3-rd Edition of the IEEE/IACSIT, ICMERA 2012, Bucharest, 26-28 October 2012, pp. 8, Invited Paper.

[19] L. Vladareanu, coordiner project et. al., "Versatile Intelligent Portable Robot Platform using Adaptive Networked Control Systems of Rescue Robots, VIPRO Platform," Project no. 009/2014 PN-II-PTPCCA-2013-4, MEN-UEFISCDI, RO, 2014-2017.

[20] L. Vladareanu, "Istoria și filosofia roboților umanoizi," Studii şi Comunicări, DIS. Vol. IX, 2016a.

[21] L. Vladareanu, M. Iliescu, H. Wang, F. Yongfei, V. Vladareanu, H. Yu, F. Smarandache, "CSP and "omics" Technology Apllied on Versatile and Intelligent Portable Platform for Modeling Complex Biomedical Data," International Conference on Advanced Mechatronic Systems (ICAMechS), pp. 423 - 428, IEEE Conferences, 2016.

[22] V. Vladareanu, I. Dumitrache, L. Vladareanu, S. I. Sacala, G. Tonț, M. A. Moisescu, "Versatile Intelligent Portable Robot Platform applied to dynamic control of the walking robots," Studies in Infor.\&Control, 24(4), pp. 409-418, ISSN:1220 - 1766, 2015.

[23] X. Wu, W. Chen, F. Ji, J. Ye, "Implementation of Robot NAO on Sprint," Journal of Computers, vol. 29, no. 2, pp. 268-275, doi:10.3966/199115992018042902026, 2018.

[24] W. Yang "Neural oscillator network-based biped control for self-adapting gait," Advances in Mechanical Engineering, vol. 10(11), pp. 1-12, DOI: 10.1177/1687814018811245, 2018. 
[25] Q. Yuan, Z. Xi, Q. Lu, Z. Lin, "Method and Experiment of the NAO Humanoid Robot Walking on a Slope Based on CoM Motion Estimation and Control," in Y. Huang et al. (Eds.), ICIRA 2017, Part III, LNAI 10464, pp. 154-165, DOI: 10.1007/978-3-319-65298-6_15, 2017.

[26] H. Zhu, M. Luo, J. Zhao, L. Lu, T. Li, "An extended landing force control method for biped robot walking on the slope," IEEE/SICE International Symposium on System Integration (SII), DOI: 10.1109/SII.2016.7843976, 2016. 